\title{
Possible consequences of applying guidelines to healthy women with a family history of breast cancer
}

\author{
CJ van Asperen ${ }^{\star, 1}$, RAEM Tollenaar ${ }^{2}$, EMM Krol-Warmerdam ${ }^{1,2}, \mathrm{~J} \mathrm{Blom}^{3}$, \\ WE Hoogendoorn ${ }^{4}$, CMJC Seynaeve ${ }^{3}$, CTM Brekelmans ${ }^{3}$, P Devilee $^{5,6}$, CJ Cornelisse ${ }^{6}$, \\ JGM Klijn ${ }^{3}$ and GH de Bock ${ }^{4}$
}

\begin{abstract}
${ }^{1}$ Center for Human and Clinical Genetics, Department of Clinical Genetics, Leiden University Medical Center, the Netherlands; ${ }^{2}$ Department of Surgery, Leiden University Medical Center, the Netherlands; ${ }^{3}$ Department of Medical Oncology, Erasmus University Medical Center Daniel den Hoed, Rotterdam, the Netherlands; ${ }^{4}$ Department of Medical Decision Making, Leiden University Medical Center, the Netherlands; ${ }^{5}$ Center for Human and Clinical Genetics, Department of Human Genetics, Leiden University Medical Center, the Netherlands; ${ }^{6}$ Department of Pathology, Leiden University Medical Center, the Netherlands
\end{abstract}

Possible effects of consistently applying published guidelines on healthy women with breast cancer in their family history were analysed. We investigated 1060 unrelated breast cancer patients and calculated the numbers of first-degree relatives that would be referred to a familial cancer clinic if the guidelines were consistently applied. A first-degree relative was considered a candidate for referral if she was female, without breast cancer at the moment of the interview, alive and over the age of 24 . The criteria for referral were based on one Dutch and two British guidelines. According to the Dutch guideline, for one affected woman with breast cancer, $0.25(95 \% \mathrm{Cl} 0.22-0.28)$ healthy first-degree female relatives should be offered a consultation at a familial cancer clinic (FCC). Application of the British guidelines would lead to a similar number of referrals. Of all healthy first-degree female relatives, who should be referred to an FCC, 34-37\% had an index case among their family who was already known at a genetic department. If current guidelines are consistently applied, a sharp increase in referrals to FCCs may be expected. These guidelines, however, are arbitrary and only limited data are available on the efficacy of this surveillance for high-risk healthy women.

European Journal of Human Genetics (2003) 11, 633-636. doi:10.1038/sj.ejhg.5201021

Keywords: guidelines; referral; familial breast cancer; familial cancer clinic; consequences

\section{Introduction}

Familial breast cancer has been a topical subject in medicine over the last years. In many studies an increased risk of developing breast cancer has been found for firstand second-degree relatives of breast cancer patients. ${ }^{1}$ Since the identification of breast cancer genes the referrals to genetic departments have been increasing rapidly in all developed countries. ${ }^{2}$

*Correspondence: $\mathrm{Dr} \mathrm{CJ}$ van Asperen, Center of Human and Clinical Genetics, Department of Clinical Genetics, Leiden University Medical Center, K5-R, PO Box 9600, 2300 RC Leiden, the Netherlands. Tel: +31 71 526 8033; Fax: +31 71526 6749; E-mail: asperen@lumc.nl Received 20 August 2002; revised 18 March 2003; accepted 1 April 2003
Management of healthy women from breast cancer families is based on individual risk assessment. Several guidelines regarding genetic risk predisposition in these women have been developed and published to guide clinicians in their approach. They are usually based on the Claus tables. ${ }^{3-7}$ The aim of these guidelines, which yield lifetime risks of developing breast cancer, is to decide whether or not a referral for a woman is indicated.

According to a guideline published by Hoskins et $a l^{8}$ a lifetime risk of $30 \%$ or more of developing breast cancer is considered an indication for referral to a familial cancer clinic (FCC). In the Netherlands, as well as in the UK, general practioners are the gatekeepers of the health care 
system, so they will refer a patient to an FCC. After such a referral further risk-assessment is done by a clinical geneticist, and, in consultation with the woman, breast and/or ovarian surveillance is taken over by the FCC. In the Netherlands there are 10 specialised FCCs, of which eight are located in university hospitals and two in cancer institutes. The American Society of Clinical Oncology (ASCO) recommend to offer DNA mutation analysis for BRCA1 and BRCA2 if the mutation detection rate is about $10 \%$; this recommendation is followed in the Netherlands. ${ }^{9}$

The objective of this paper is to study the potential effect of consistently applying sets of referral criteria to healthy female first-degree relatives of breast cancer patients on the number of referrals to familial cancer clinics. Three current guidelines for referral to familial cancer clinics were compared; one Dutch and two British, which are widely used by clinicians involved in the care of breast cancer patients and their families. ${ }^{5-7}$

\section{Methods}

Patients with a recent diagnosis of breast cancer were asked to participate in the ongoing 'PROSPECT cohort'. This study was carried out in the SouthWest area of the Netherlands (in two academic centres in Leiden and Rotterdam and in one general hospital in Leiden) between 1 November, 1997 and 1 July, 2002. Family histories were collected from 1154 patients. In this analysis only patients with a diagnosis of breast cancer under the age of 70 years were included ( $n=1060,388$ from the Leiden area and 672 from the Rotterdam area). The medical ethics committees of the three hospitals approved the study protocol for the 'PROSPECT cohort'. To obtain a family history of all types of cancer in first- and second-degree relatives, an interview and a questionnaire were part of the procedure. Pedigrees were drawn and included in a Cyrillic 2.0 database. Brothers, sisters, parents and children of this patient were regarded as first-degree relatives and paternal and maternal aunts and uncles, paternal and maternal grandparents and grandchildren as second-degree relatives. Information on the number of index patients already known at the genetic departments was also collected.

The numbers of potential referrals were calculated for first-degree relatives. A first-degree relative was considered a potential client for genetic counselling if she was female, without breast cancer at the moment of the interview, alive and over 24 years of age. The age of 25 was chosen as starting point for breast surveillance in high-risk families. ${ }^{10}$ The total number of referrals to an FCC was calculated for the three guidelines; NL CBO, UK CSFG and UK BASO. ${ }^{5-7}$ In Table 1 , the referral criteria of these three guidelines are given. The mean number of referrals per index case of breast cancer as well as the 95\% confidence intervals (CI) were computed. Additionally, the percentage of agreement between the guidelines was assessed. The number of index cases who were already known at a genetic department was
Table 1 Reasons for referral to a familial cancer clinic according to three guidelines

$\mathrm{NL} \mathrm{CBO}{ }^{5}$

- $\geqslant 2$ first-degree relatives with breast cancer <age 50 years

- $\geqslant 3$ first-degree relatives with breast cancer $\geqslant$ age 50 years

- $\geqslant 2$ second-degree relatives with breast cancer < age 50 years

- $\geqslant 3$ second-degree relatives with breast cancer $\geqslant$ age 50 years

- Breast cancer and prostate cancer <age 60 in one family

- Breast cancer and ovarian cancer in one family

UK CFSG ${ }^{6}$

- Two relatives with breast cancer average age 30-39 years

- Three relatives with breast cancer average age 40-50 years

- Three relatives with breast cancer average age 50-60 years breast and other cancers:

- $\geqslant 1$ relative with breast cancer $\leqslant$ age 50 years $+\geqslant 1$ relative with ovarian cancer at any age or 1 relative with both.

- $\geqslant 1$ relative with breast cancer < age 40 years + relative with childhood malignancy

UK BASO ${ }^{7}$

- Breast/ovarian families with $\geqslant 4$ relatives on the same side of the family affected, at any age

- Breast cancer (only) families with three affected relatives with an average age at diagnosis of age $<40$ years

- Breast/ovarian families with three affected relatives with an average age at diagnosis of breast cancer $<60$ years

- Families with one member with both breast and ovarian cancer

assessed as well. Their first-degree female relatives were excluded and the data were analysed again. The mean number of referrals per index case of breast cancer as well as the $95 \%$ CI were computed.

This analysis was also repeated after excluding index patients with a diagnosis of ductal carcinoma in situ (DCIS).

To determine whether this study cohort of breast cancer patients was representative for the Dutch female population of women diagnosed with breast cancer, regarding age at diagnosis, a comparison was made with a cohort taken from the Comprehensive Cancer Centre West over the years 1996-2000. Because distribution of the latter cohort was given as a categorical variable, $\chi^{2}$ was calculated. Furthermore, a comparison of the family histories was made with earlier reports of population-based studies. ${ }^{11,12}$ Statistical analysis was performed with SPSS 11.01.

\section{Results}

In 1060 unrelated family histories of women with breast cancer 29037 individuals were reported. These families included 3830 first-degree female relatives of whom 2554 (67\%) were alive, without breast cancer at the moment of the interview and above the age of 24 years. The referrals to an FCC were estimated in the group of 2554 healthy firstdegree female relatives of the 1060 recently diagnosed breast cancer patients according to the three guidelines. According to the NL CBO guideline, 264 women should be referred to an FCC. For the UK CFSG guideline this was estimated at 296 women and for the BASO guideline at 264 women. 
The mean number of healthy first-degree female relatives referred per index case of breast cancer was calculated. Application of the Dutch guideline led to 0.25 (95\% CI $0.22-0.28$ ) referrals per case of breast cancer. Application of the British guidelines in this group led to 0.28 (95\% CI $0.25-$ $0.31)$ and 0.25 (95\% CI $0.22-0.28)$ referral per index case.

The Dutch guideline had a percentage of agreement of 90 with both UK guidelines $(\kappa=0.57 ; P<0.001$ and $\kappa=0.55$; $P<0.001$ for comparison with UK CFSG and BASO, respectively). The percentage of agreement between the UK guidelines was $97(\kappa=0.89 ; P<0.001)$.

Based on the Dutch guideline, 36\% (95\% CI 33-39) of the first-degree females who ought to get a referral to an FCC had an index family member who was already known at a genetic department. For the UKCFSG and the BASO, this percentage was 34 (95\% CI 31-37) and 37 (95\% CI 3440), respectively.

DCIS was diagnosed in $15 \%(n=156)$ of the index cases in our study. After exclusion of DCIS, comparable referral rates were found as in the first analyses in which DCIS was included (data not shown).

In the study cohort, breast cancer was diagnosed in $9 \%$ of the cases before the age of 40 years, in $28 \%$ between the age of 40 and 50 years, in $38 \%$ between the age of 50 and 60 years and in $25 \%$ between the age of 60 and 70 years. The distribution of age of the index cases was similar to the cohort of the Comprehensive Cancer Centre West. Of the 3880 women diagnosed in the latter cohort with breast cancer, $9 \%$ were under the age of 40 years, $28 \%$ between the age of 40 and 50 years, $35 \%$ between the age of 50 and 60 years and $28 \%$ between the age of 60 and 70 years $\left(\chi^{2}=0.29 ; \mathrm{df}=3 ; P=0.96\right)$.

The percentage of index cases with at least a sister affected by breast cancer was $8.6 \%$ (95\% CI 6.9-10.3) in our study. In a British population study of 1323 incident cases of breast cancer diagnosed under the age of 65 years, $5 \%$ (95\% CI 3.8-6.2) of the women had a sister with breast cancer. ${ }^{11}$ According to the Nurses' Health Study of 2318 incident cases of breast cancer under the age of 55 years, $4.1 \%$ (95\% CI 3.3-4.9) of the women had a sister with breast cancer. $^{12}$ The percentage of index cases with a mother affected by breast cancer was $8.8 \%$ (95\% CI 6.8-10.8) in our study and 11\% (95\% CI 9.3-12.8) and $10.3 \%$ (95\% CI 9.0-11.6) in the two population studies, respectively. ${ }^{11,12}$

\section{Discussion}

The results of this study indicate that according to three current guidelines, one female first-degree relative in every four newly diagnosed breast cancer patients should be referred to a familial cancer clinic. In this hospital-based cohort of recently diagnosed breast cancer patients, roughly two-thirds of the first-degree relatives in the families seem to have been unknown at an FCC.
The study cohort on which the data are based is representative of women with breast cancer in the Netherlands according to their age at onset. Regarding the family histories, it could be considered that these data are roughly comparable to earlier reports of population-based studies, although the percentage of affected sisters in the presented study is higher and the percentage of affected mothers is lower. The higher percentage of affected sisters could be explained by the younger ages of the incident cases in the population studies. ${ }^{11,12}$

In this study, DCIS (15\% of the index cases) was regarded as breast carcinoma. The referral rate for healthy firstdegree female relatives was the same when DCIS were excluded. However, only index cases with DCIS were excluded for this additional analysis because pathology reports concerning breast tumours of other family members were not presented. This may overestimate the number of referrals, but on the other hand this may be comparable with everyday practice. Many relatives with DCIS will be included when considering an affected family history for breast cancer. Clinicians applying guidelines to healthy women with breast cancer in their family history often include women who were diagnosed with DCIS as well. Pathology reports of family members are usually not available and healthy family members mostly consider DCIS as an invasive breast carcinoma. Moreover, DCIS may turn into an invasive breast carcinoma.

Although there is already an increased public demand for information reassurance and cancer screening, it seems that more and more referrals have to be expected based on the results of this study. According to two British studies, an increase of referrals to an FCC has already been seen as GPs are becoming more acquainted with these guidelines. ${ }^{13,14}$

More referrals to an FCC will result in an increase in the use of health care facilities. There will not only be a consultation with a clinical geneticist, but if breast surveillance is indicated, regular visits to a physician combined with mammograms will follow. MRI scans are momentarily offered in a research setting. In general, referrals to the gynaecologist for ovarian surveillance are offered as well if a BRCA1 of BRCA2 mutation is detected in the family, or if a relative has been diagnosed with ovarian cancer. ${ }^{15}$ It is obvious that these additional FCC referrals to surgeons, gynaecologists and radiologists are increasing rapidly, more so because FCC referrals for breast and ovarian surveillance have a lifelong character. One may wonder if this lifelong character is justified. As far as we know, there are no evidenced-based data on the desirability of an upper age limit for breast and/or ovarian surveillance. As the known predisposition genes for breast cancer, including BRCA1 and BRCA2, have been estimated to account for only $20-25 \%$ of familial aggregation, ${ }^{16}$ the number of healthy women, who may be discharged from breast and ovarian surveillance, based on a negative presymptomatic DNA test result, is also limited. 
Current breast surveillance in BRCA1 and BRCA2 families consists of physical examination and mammography and has low sensitivity. ${ }^{17,18}$ Inclusion of a breast MRI in breast surveillance programs of healthy high-risk women might lead to a higher sensitivity and will probably result in a lower mortality rate. ${ }^{19,20}$ No evidence has been found so far for ovarian surveillance as an effective way to reduce mortality. ${ }^{21}$

Based on the presented data, it is not possible to give an exact population-based referral rate for FCCs for healthy women in the Netherlands. Every year, approximately 10000 women in the Netherlands are diagnosed with breast cancer. ${ }^{22}$ In this study, the families are unrelated. In the general population there might be an increasing degree of relation, which yields overestimation. A model of potential demand would need to integrate not only incident cases, as in this presented study, but also prevalent ones. In addition, with the unknown numbers of already counted family members in former years, it will be impossible to predict the exact referral numbers for the coming years. Moreover, in the Netherlands and the UK general practitioners are the gatekeepers of the health care system, which is probably not the case in other European countries. In these countries, guidelines might be used directly by clinical geneticists or surgeons, in order to justify further surveillance.

Nevertheless it is clear that, if current guidelines for healthy relatives of breast cancer patients are consistently applied by all clinicians involved in the care of breast cancer patients and their families, a sharp increase in the use of genetic services, surgical and gynaecological consultations, as well as breast and ovarian imaging procedures is to be expected.

Scientific advancement in breast cancer genetics combined with a growing public interest in breast cancer prevention could be hindered by economic limitations. The criteria for the management of healthy women from breast cancer families are arbitrary and not yet evidencebased. Only limited data are available on the efficacy of surveillance. For that reason, surveillance of healthy women with a high cumulative risk of developing breast cancer should be included in a prospective study design. Thus, it may be assessed which groups of women will really benefit from surveillance.

\section{Acknowledgements}

The authors thank the surgeons and mamma care nurses of the Diaconessen Hospital Leiden and the referring specialists in both academic hospitals for their contribution.

This work was supported by the Dutch Cancer Society (Grant RUL 97-1505).

\section{References}

1 Pharoah PD, Day NE, Duffy S, Easton DF, Ponder BA: Family history and the risk of breast cancer: a systematic review and meta-analysis. Int J Cancer 1997; 71: 800-809.
2 Hodgson SV, Haites NE, with BIOMED II partners: A survey of the current clinical facilities for the management of familial cancer in Europe. J Med Genet 2000; 37: 605-607.

3 Claus EB, Risch N, Thompson WD: Autosomal dominant inheritance of early onset breast cancer. Cancer 1994; 73: 643-651.

4 de Bock GH, Vliet Vlieland TP, Hageman GC, Oosterwijk JC, Springer MP, Kievit J: The assessment of genetic risk of breast cancer: a set of GP guidelines. Fam Pract 1999; 16: 71-77.

5 van Asperen CJ, de Bock GH, van der Horst F, de Koning HJ, Rutgers EJ: Guidelines for breast cancer surveillance for women not eligible for population screening, by individual risk assessment. Ned Tijdschr Geneeskd 2001; 145: 120-125.

6 Eccles DM, Evans DG, Mackay J: Guidelines for a genetic risk based approach to advising women with a family history of breast cancer. UK Cancer Family Study Group (UKCFSG). J Med Genet 2000; 37: 203-209.

7 Blamey RW: The British Association of Surgical Oncology Guidelines for surgeons in the management of symptomatic breast disease in the UK (1998 revision). BASO Breast Specialty Group. Eur J Surg Oncol 1998; 24: 464-476.

8 Hoskins KF, Stopfer JE, Calzone KA et al: Assessment and counseling for women with a family history of breast cancer. JAMA 1995; 273: 577-585.

9 Statement of the American Society of Clinical Oncology: genetic testing for cancer susceptibility, Adopted on February 20, 1996. J Clin Oncol 1996; 14: 1730-1736.

10 Burke W, Daly M, Garber J, Botkin J et al: Recommendations for follow-up care of individuals with an inherited predisposition to cancer. II. BRCA1 and BRCA2. Cancer Genetics Studies Consortium. JAMA 1997; 277: 997-1003.

11 Pharoah PD, Lipscombe JM, Redman KL, Day NE, Easton DF, Ponder BA: Familial predisposition to breast cancer in a British population: implications for prevention. Eur J Cancer 2000; 36: 773-779.

12 Colditz GA, Willett WC, Hunter DJ et al: Family history, age, and risk of breast cancer, Prospective data from the Nurses' Health Study. JAMA 1993; 270: 338-343.

13 Lucassen A, Watson E, Harcourt J, Rose P, O'Grady J: Guidelines for referral to a regional genetics service: GPs respond by referring more appropriate cases. Fam Pract 2001; 18: 135-140.

14 Wonderling D, Hopwood P, Cull A et al: A descriptive study of UK cancer genetics services: an emerging clinical response to the new genetics. Br J Cancer 2001; 85: 166-170.

15 Vasen HF, Haites NE, Evans DG et al: Current policies for surveillance and management in women at risk of breast and ovarian cancer: a survey among 16 European family cancer clinics. European Familial Breast Cancer Collaborative Group. Eur J Cancer 1998; 34: 1922-1926.

16 Easton DF: How many more breast cancer predisposition genes are there? Breast Cancer Res 1999; 1: 14-17.

17 Brekelmans CT, Seynaeve C, Bartels CCM et al: Effectiveness of breast cancer surveillance in BRCA1/2 gene mutation carriers and women with high familial risk. J Clin Oncol 2001; 19: $924-30$.

18 Meijers-Heijboer H, van Geel B, van Putten WL et al: Breast cancer after prophylactic bilateral mastectomy in women with a BRCA1 or BRCA2 mutation. $N$ Engl J Med 2001; 345: 159-164.

19 Kuhl CK, Schmutzler RK, Leutner CC et al: Breast MR imaging screening in 192 women proved or suspected to be carriers of a breast cancer susceptibility gene: preliminary results. Radiology 2000; 215: 267-279.

20 Stoutjesdijk MJ, Boetes C, Jager GJ et al: Magnetic resonance imaging and mammography in women with a hereditary risk of breast cancer. J Natl Cancer Inst 2001; 93: 1095-1102.

21 Hallowell N, Jacobs I, Richards M, Mackay J, Gore M: Surveillance or surgery? A description of the factors that influence high risk premenopausal women's decisions about prophylactic oophorectomy. J Med Genet 2001; 38: 683-691.

22 Visser O, Coebergh JWW, Schouten LJ et al: Incidence of cancer in the Netherlands. Comprehensive Cancer Centers, Utrecht, 2001. 